\title{
Risk factors related to the recurrence of endometrioma in patients with long-term postoperative medical therapy
}

\author{
Sieun Han, Hyunjoo Lee, Seungchul Kim, Jongkil Joo, Dongsoo Suh, Kihyung Kim, Kyusup Lee \\ Department of Obstetrics and Gynecology, Pusan National University School of Medicine Biomedical Research Institute, \\ Pusan National University Hospital, Busan, Korea
}

\begin{abstract}
Objectives: The purpose of this study was to identify clinical risk factors for the recurrence of ovarian endometrioma after ovarian cystectomy in Korean women with long-term postoperative medical therapy.

Material and Methods: A total of 134 patients who were surgically treated for endometriotic cysts at Pusan National University Hospital were included in this retrospective study. All patients received long-term postoperative medical treatment for at least 12 months after the first-line conservative surgery. Several epidemiologic variables were analyzed as possible risk factors for recurrence. Endometrioma recurrence was considered when a cystic mass was observed on transvaginal or transrectal sonography. Statistical analysis was performed using independent t-tests for parametric continuous variables. Results: The mean follow-up period for the 134 patients was $56.5 \pm 14.3$ months (range, $36-120$ months) and the mean duration of the medical therapy was $17.9 \pm 17.3$ months (range, $12-120$ months). The overall recurrence rate was 35/134 (26.12\%). Our univariate analysis showed statistically significant differences between the recurrent and non-recurrent groups in terms of weight $(P=0.013)$, body mass index $(P=0.007)$, age at the time of surgery $(P=0.013)$, the diameter of the largest cyst $(P=0.001)$, the presence of dysmenorrhea $(P<0.0001)$, and postoperative pregnancy $(P=0.016)$. Multivariate analysis showed that body mass index (OR $1.153,95 \% \mathrm{Cl} 1.003-1.326, \mathrm{P}=0.046)$, age at the time of surgery $(\mathrm{OR} 0.924,95 \% \mathrm{Cl}$ $0.860-0.992, P=0.029$ ), and presence of dysmenorrhea (OR 12.226, 95\% Cl 3.543-42.188, $\mathrm{P}<0.0001$ ) were significantly correlated with the recurrence of endometrioma.

Conclusions: We found that patients with dysmenorrhea after surgery, and a younger age of the patient at the time of surgery were the highest risk factors associated with the recurrence of endometrioma, despite long-term postoperative medication. Key words: endometrioma; recurrence rate; cystectomy; endometriosis
\end{abstract}

Ginekologia Polska 2018; 89, 11: 611-617

\section{INTRODUCTION}

Endometriosis is characterized by the growth of endometrial tissue outside the uterine cavity, which induces a chronic inflammatory reaction [1]. Ovarian endometrioma is observed in approximately $20-40 \%$ of women with endometriosis [2]. Endometriosis is predominant in women of reproductive age with a prevalence of 10 to $15 \%[3,4]$. Several theories for this have been suggested, including retrograde menstruation, altered immunity, coelomic metaplasia, and metastatic spread. However, the pathogenesis of endometriosis is still unknown. [5]. The treatment of choice for endometrioma is usually surgery because of the limited ef- ficacy of medication and the possibility of malignancy [6, 7]. However, vigorous debates about surgical approaches to the treatment of ovarian endometrioma in relation to ovarian reserve are ongoing [8].

The recurrence of endometrioma is one of the most important unresolved problems in the management of endometriosis. There have been two hypotheses that seek to explain the underlying pathophysiology of endometrioma recurrence: growth from residual lesions, or the development of retrograde menstruation after surgery [9].

Recently, Seo et al. suggested the young age of women undergoing treatment as a risk factor for endometrioma recur- 
rence [10]. Moreover, there are some studies on risk factors for recurrence. The patient's age, postoperative pregnancy, the severity of endometriosis and the size of the ovarian cyst were proposed as related factors in the recurrence of endometrioma among Japanese women [11-14]. However, there are only a few studies conducted in Korea. Lee et al. reported the risk factors associated with recurrence of endometrioma in patients younger than 20 years; and Kim et al. reported risk factors in patients with a history of two or more previous surgical managements for endometriosis $[15,16]$. However, these studies included heterogeneous patient groups, which combined all the patients with or without postoperative medical therapy.

Because endometrioma is widely reported to have a high recurrence rate after surgery, some clinicians prefer to prescribe long-term use of oral contraceptives or progestin in order to prevent recurrence, and long-term postoperative medication has become the standard therapy for the women who wish to conceive in the future [17]. Therefore, it would be valuable to investigate the risk factors that are related to endometrioma recurrence in patients receiving long-term medical therapy.

The purpose of our study was to analyze the risk factors associated with endometrioma recurrence among Korean patients receiving long-term medical therapy to prevent recurrence.

\section{MATERIAL AND METHODS}

\section{Patients}

Our study was a retrospective analysis of the records of patients with an histologic diagnosis of endometrioma who had long-term medical therapy. The study group was comprised of 134 patients selected from 334 patients who had been surgically treated by a single surgeon at Pusan National University Hospital between January 2008 and December 2014. Patients with the following histories were included in this study: 1). surgical treatment of endometriotic cysts; 2). final diagnosis of endometriosis confirmed by pathological findings; and 3). postoperative medical treatment for at least 12 months. Exclusion criteria were as follows: 1). discontinuation of medical treatment for a period of more than one month for any reason; 2). previous hysterectomy and/or bilateral oophorectomy for therapeutic purpose for endometriosis or any other illness; 3). diagnosis of menopause, post-operatively or during follow-ups; and 4). any medical history that could influence medication metabolism. The patients underwent clinical postoperative follow-ups at intervals of three to six months. In each follow-up visit, clinical data were recorded after a transvaginal ultrasound, pelvic examination and serum CA-125 test.

\section{Endometrioma recurrence}

All patients had a transvaginal ultrasound scan administered by an experienced sonographer and using the Interna- tional Ovarian Tumor Analysis (IOTA) terms and definitions to describe the ultrasound findings [18]. Endometrioma recurrence was defined by typical features, such as the presence of a ground glass-shaped cyst with a homogenous low-level echogenicity.

\section{Epidemiological and clinical risk factors}

Medical chart reviews and patients' interview records were used to collect patient information and calculate demographics such as weight, height, body mass index (BMI), parity, age at the time of surgery, previous history of cesarean section, number of previous surgeries, time to recurrence, unilateral or bilateral involvement, presence of cul-de-sac adhesions, the diameter of the largest cyst, the type of surgical management (laparotomy or laparoscopy), postoperative medication, the presence of dysmenorrhea, postoperative pregnancy in patients with postoperative medication of 12 months of more, total revised ASRM scores, and stage of the disease. The stage of the disease was defined according to the revised American Society for Reproductive Medicine (rASRM) scale as stage I (minimal), stage II (mild), stage III (moderate), and stage IV (severe) [19].

\section{Statistical analysis}

Comparisons of continuous data between two groups were performed with the use of an independent t-test or Wilcoxon rank-sum test (Mann-Whitney-Wilcoxon test), and categorical data were analyzed using the chi-square test or Fisher's exact test. Univariate and multivariate analyses were performed using multiple logistic regression analysis, to evaluate the possible risk factors for endometrial cyst recurrence. Statistical analysis was performed using SAS 9.3 for Windows (SAS Institute, Cary, NC, USA). Statistical significance was set at $P<0.05$.

\section{RESULTS}

The mean follow-up period for the one hundred and thirty-four patients in our study was 56.5 months (range, 36-120 months), and the median duration of medical therapy was 12 months (range, 12-120 months). The clinical characteristics of the patients with, and without, the recurrence of endometrioma are shown in Table 1. From a total of 134 consecutive patients who were treated with laparoscopy and/or laparotomy, endometrioma recurred in 35 patients, and the overall recurrence rate was $26.1 \%$.

Weight, BMI, age at the time of surgery, size of endometrioma, rASRM score and stage, the presence of dysmenorrhea after surgery, and postoperative pregnancy with postoperative medication of 12 months or more, each proved to have a statistically significant association with the recurrence of endometrioma. Fifteen of the 134 women became pregnant after long-term medication, and endometrioma recurrence was observed in eight of those patients. Furthermore, five patients out of those eight patients diagnosed with 
Table 1. Characteristics of patients with and without the recurrence of ovarian endometrioma and $P$ values from the univariable analysis

\begin{tabular}{|c|c|c|c|c|c|}
\hline & & Total $(n=134)$ & Non-recurrent group $(n=99)$ & Recurrent group $(n=35)$ & P-value* \\
\hline Weight [Kg] & & $57.44(9.07)$ & $56.23(7.98)$ & $60.84(11.04)$ & 0.028 \\
\hline Height [meter] & & $1.62(0.05)$ & $1.62(0.05)$ & $1.61(0.05)$ & 0.466 \\
\hline BMI [kg/m2] & & $22.01(3.56)$ & $21.48(3.03)$ & $23.49(4.47)$ & 0.018 \\
\hline \multirow[t]{5}{*}{ Parity**,$\neq$} & 0 & $100(74.63)$ & $71(71.72)$ & $29(82.86)$ & 0.447 \\
\hline & 1 & $14(10.45)$ & $13(13.13)$ & $1(2.86)$ & \\
\hline & 2 & $18(13.43)$ & $13(13.13)$ & $5(14.29)$ & \\
\hline & 3 & $1(0.75)$ & $1(1.01)$ & $0(0.00)$ & \\
\hline & 4 & $1(0.75)$ & $1(1.01)$ & $0(0.00)$ & \\
\hline Age at the time of surgery & & $34.23(8.08)$ & $35.28(8.35)$ & $31.26(6.49)$ & 0.011 \\
\hline \multirow[t]{3}{*}{ Bilaterality** } & Left & $40(29.85)$ & $30(30.30)$ & $10(28.57)$ & 0.640 \\
\hline & Right & $45(33.58)$ & $35(35.35)$ & $10(28.57)$ & \\
\hline & Both & $49(36.57)$ & $34(34.34)$ & $15(42.86)$ & \\
\hline \multirow[t]{2}{*}{ CDS obliteration** } & No & $62(46.27)$ & $43(43.43)$ & $19(54.29)$ & 0.268 \\
\hline & Yes & $72(53.73)$ & $56(56.57)$ & $16(45.71)$ & \\
\hline \multirow[t]{2}{*}{ Size ${ }^{* *}$} & $0-3 \mathrm{~cm}$ & $58(43.28)$ & $52(52.53)$ & $6(17.14)$ & $<0.001$ \\
\hline & $>3 \mathrm{~cm}$ & $76(56.72)$ & $47(47.47)$ & $29(82.86)$ & \\
\hline rASRM score ${ }^{*}, \dagger$ & & $44.50[28.00,84.00]$ & $52.00[31.00,93.00]$ & $41.00(20.00,76.00)$ & 0.048 \\
\hline \multirow[t]{4}{*}{ rASRM stage ${ }^{* *}, \neq$} & 1 & $6(4.48)$ & $3(3.03)$ & $3(8.57)$ & 0.017 \\
\hline & 2 & $5(3.73)$ & $1(1.01)$ & $4(11.43)$ & \\
\hline & 3 & $47(35.07)$ & $38(38.38)$ & $9(25.71)$ & \\
\hline & 4 & $76(56.72)$ & $57(57.58)$ & $19(54.29)$ & \\
\hline \multirow[t]{2}{*}{ Type of surgery** } & Laparotomy & $53(39.55)$ & $42(42.42)$ & $11(31.43)$ & 0.253 \\
\hline & Laparoscopy & $81(60.45)$ & $57(57.58)$ & $24(68.57)$ & \\
\hline \multirow[t]{2}{*}{ Dysmenorrhea** } & No & $111(82.84)$ & $93(93.94)$ & $18(51.43)$ & $<0.0001$ \\
\hline & Yes & $23(17.16)$ & $6(6.06)$ & $17(48.57)$ & \\
\hline \multirow[t]{2}{*}{ Postoperative pregnancy ${ }^{* *}, \neq$} & No & $119(88.81)$ & $92(92.93)$ & $27(77.14)$ & 0.024 \\
\hline & Yes & $15(11.19)$ & $7(7.07)$ & $8(22.86)$ & \\
\hline Postoperative CA-125 $(\mathrm{U} / \mathrm{mL}) *$, $†$ & & $16.07[11.90,29.43]$ & $15.00[10.73,22.79]$ & $24.95(14.64,55.40)$ & 0.007 \\
\hline
\end{tabular}

Independent t-test or Wilcoxon rank-sum test $(\dagger)$ for continuous variables; Chi-square test or Fisher's exact test ( $\neq$ ) for categorical variables; Values are mean (standard deviation); ${ }^{*}$, median (min-max); ${ }^{* *}$, number [\%]; BMI — body mass index; rASRM — revised American Society for Reproductive Medicine

recurrent endometrioma had undergone cesarean section. In our study, the serum CA-125 level was higher in patients with recurrent endometrioma (median level $24.95 \mathrm{U} / \mathrm{mL}$ ) compared with those without recurrent endometrioma (median level $15 \mathrm{U} / \mathrm{mL}$ ). However, our univariate analysis showed that the recurrence risk was not significantly associated with the postoperative serum CA-125 level.

The relative risks of recurrence of endometrioma revealed in the results of our univariate analysis shown in Table 2. In the univariate analysis, the risk of recurrence was significantly affected by weight, BMI, age at the time of surgery, diameter of the largest cyst, presence of dysmenorrhea, and postoperative pregnancy.

The results of our multivariate logistic regression analysis are presented in Table 3. According to the multivariate analysis, the significant factors that were associated with the recurrence of endometrioma were the body mass index (OR 1.153, 95\% Cl 1.003-1.326, $P=0.046)$, age at the time of surgery (OR $0.924,95 \% \mathrm{Cl} 0.860-0.992, P=0.029$ ) and the presence of dysmenorrhea (OR 12.226, 95\% Cl 3.543-42.188, $P<0.0001)$. Across the entire cohort, the number of patients with recurrent endometrioma during the follow-up period gradually increased, reaching a total of 35 patients (26.1\%) by the end of the 5-year postoperative period (Fig. 1).

\section{DISCUSSION}

Endometriosis has been systematically investigated for decades, but its treatment efficacy and recurrent risk factors are still only vaguely understood. The aim of this study was to explore the risk factors for the recurrence of endometrioma after long-term medical therapy and to find personalized patterns according to these risk factors. In this 
Table 2. Univariate logistic regression analyses of factors related to endometrioma recurrence

\begin{tabular}{|c|c|c|c|c|c|}
\hline \multirow{2}{*}{ Predictors } & & \multirow{2}{*}{ OR } & \multicolumn{2}{|l|}{$95 \% \mathrm{Cl}$} & \multirow{2}{*}{ P-value } \\
\hline & & & Lower & Upper & \\
\hline Weight [Kg] & & 1.056 & 1.011 & 1.102 & 0.013 \\
\hline Height [meter] & & 0.971 & 0.899 & 1.050 & 0.463 \\
\hline BMI [kg/m2] & & 1.164 & 1.043 & 1.299 & 0.007 \\
\hline Parity $(0) \dagger$ & & 0.773 & 0.452 & 1.321 & 0.346 \\
\hline Age at the time of surgery & & 0.934 & 0.885 & 0.986 & 0.013 \\
\hline Extremity surgery & & 0.779 & 0.222 & 2.734 & 0.696 \\
\hline Abdominal surgery & & 0.539 & 0.112 & 2.592 & 0.441 \\
\hline Gynecologic surgery & & 1.463 & 0.616 & 3.475 & 0.389 \\
\hline Previous cesarean section & & 0.967 & 0.529 & 1.769 & 0.914 \\
\hline Previous surgeries & & 0.985 & 0.645 & 1.503 & 0.944 \\
\hline rASRM score (per 10 points) $†$ & & 0.988 & 0.977 & 1.000 & 0.053 \\
\hline \multirow[t]{2}{*}{ Bilaterality (Left) † } & Right & 0.857 & 0.314 & 2.337 & 0.763 \\
\hline & Both & 1.324 & 0.518 & 3.384 & 0.558 \\
\hline CDS obliteration (No) $†$ & Yes & 0.647 & 0.298 & 1.403 & 0.270 \\
\hline Size $(0-3 \mathrm{~cm}) \dagger$ & $>3 \mathrm{~cm}$ & 5.348 & 2.040 & 14.015 & 0.001 \\
\hline \multirow[t]{3}{*}{ rASRM stage (1) $\dagger$} & 2 & 3.997 & 0.265 & 60.260 & 0.317 \\
\hline & 3 & 0.237 & 0.041 & 1.373 & 0.108 \\
\hline & 4 & 0.333 & 0.062 & 1.793 & 0.201 \\
\hline Type of surgery (Laparotomy) † & Laparoscopy & 1.607 & 0.710 & 3.640 & 0.255 \\
\hline Dysmenorrhea (No) † & Yes & 14.639 & 5.078 & 42.200 & $<0.0001$ \\
\hline Postoperative pregnancy (No) $†$ & Yes & 3.894 & 1.294 & 11.715 & 0.016 \\
\hline Postoperative CA-125 [U/mL] & & 1.000 & 0.997 & 1.003 & 0.952 \\
\hline
\end{tabular}

†, (reference); BMI — body mass index; rASRM — revised American Society for Reproductive Medicine

Table 3. Multivariate logistic regression analyses of factors related to endometrioma recurrence

\begin{tabular}{|c|c|c|c|c|c|}
\hline \multirow{2}{*}{ Predictors } & \multirow{2}{*}{ level } & \multirow{2}{*}{ OR } & \multicolumn{2}{|l|}{$95 \% \mathrm{Cl}$} & \multirow{2}{*}{ P-value * } \\
\hline & & & Lower & Upper & \\
\hline BMI & & 1.153 & 1.003 & 1.326 & 0.046 \\
\hline Age at the time of surgery & & 0.924 & 0.860 & 0.992 & 0.029 \\
\hline Size $(0-3 \mathrm{~cm}) \dagger$ & $>3 \mathrm{~cm}$ & 2.845 & 0.940 & 8.608 & 0.064 \\
\hline Dysmenorrhea (No) † & Yes & 12.226 & 3.543 & 42.188 & $<0.0001$ \\
\hline Postoperative pregnancy $(\mathrm{No}) \dagger$ & Yes & 3.334 & 0.887 & 12.527 & 0.075 \\
\hline
\end{tabular}

$\dagger$, (reference), BMI — body mass index

retrospective study, our univariate analysis showed that the weight, BMI, age at the time of surgery, the diameter of the cyst, the presence of dysmenorrhea, and postoperative pregnancy to be strongly associated with the recurrence of endometrioma. However, our multivariate analysis showed that the size of the cyst and postoperative pregnancy were not significantly associated with recurrent endometrioma.

Some reports have indicated that the young age of patients was a risk factor [12, 20-22], while Parazzini et al. reported that higher recurrence rates were observed in older women [23]. The association between the patient's age at surgery and endometrioma recurrence has been consistently mentioned in previous studies [13,20], and our study data showed that there is a statistically significant association between younger-aged patients at the time of surgery and the risk of recurrent endometrioma. The reason for this increased recurrence rate is unclear, but we postulate that the higher circulation estrogens levels in younger women 


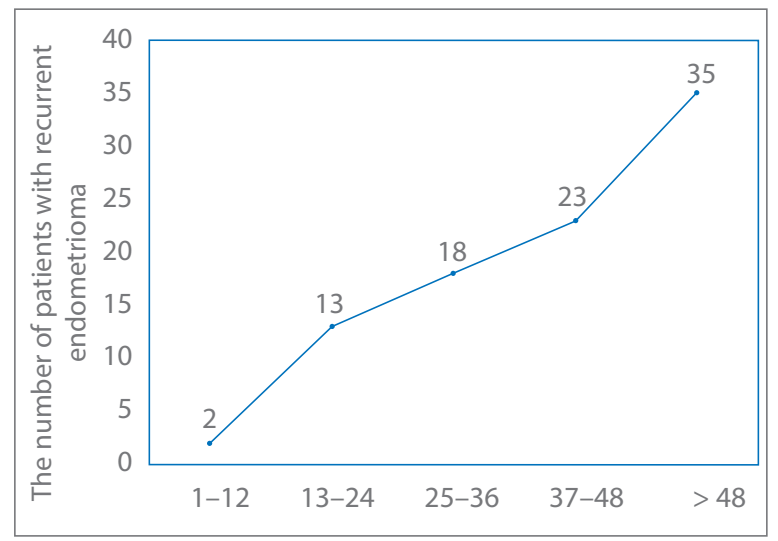

Figure 1. Estimated cumulative number of patients with a recurrence of ovarian endometrioma after laparoscopic cystectomy in Korean women who were treated with postoperative medical therapy

at the time of the endometrioma diagnosis, may produce a more aggressive form of endometriosis, therefore, a form that is more likely to recur compared with the form of endometriosis found in in older patients [11]. The patient's age at surgery may well be a surrogate marker for the circulating estrogens levels in the patient [22]. However, in this study, we did not measure the estrogens levels and this postulation should be confirmed with further prospective studies.

While high $\mathrm{BMI}$ is cited as a risk factor for endometriosis by several authors $[24,25]$, the relation is far from clear. We too found that high BMI was a significant risk factor of recurrent endometrioma. Increased body weight may mask chronic symptoms such as pain, therefore resulting in less frequent diagnosis of endometriosis. Therefore, in such occasions there is a possibility that endometriosis could be diagnosed at a more advanced stage. Also, the complete removal of ovarian endometrioma can prove challenging in obese patients.

Several studies identified dysmenorrhea as a possible risk factor for endometrioma recurrence [26, 27]. Our study also showed an association between the presence of dysmenorrhea after surgery and recurrent endometrioma. Endometriotic lesions and adhesions may also cause deep pelvic pain associated with recurrent endometriosis [27]. In conclusion, postoperative dysmenorrhea could suggest the recurrence of endometrioma.

It is clear from numerous reports that the prevalence of recurrent endometrioma varies according to whether there is a successful pregnancy after surgical treatment of the endometrioma [11, 20, 22, 28]. Koga et al. demonstrated that patients with post-operative pregnancies had much lower rates of recurrence, which indicates that a subsequent pregnancy may have a protective effect in relation to the risk of recurrence of the endometrioma [13]. However, postoperative pregnancies did not prevent the recurrence of endometrioma in our study (OR, 3.334, 95\% $\mathrm{Cl}, 0.887-12.527, P$-value $=0.075$ ). Our results may be due to the limited number of cases we studied. Another possible explanation is iatrogenic spreading of endometrial tissue to the pelvic cavity during cesarean section [29, 30]. Five patients with successful pregnancies after their initial surgery, but who later had recurrent endometrioma, were diagnosed after cesarean sections. In addition, more conservative fertility-sparing surgery to minimize the loss of healthy ovarian tissue, should be a primary concern for treating infertile women with presumed endometriosis [31], as it is technically challenging for the surgeon to completely remove ovarian endometrioma in infertile women.

Several studies found an association between caesarean section and endometriosis in the pelvic cavity [30, 32]. We performed a statistical analysis to compare the types of previous operations and their relations to endometrioma recurrence. The types of previous operations included extremity operations such as arm and leg fracture surgery, abdominal operations, and gynecologic operations other than endometriosis and/or cesarean section [30, 33]. However, in our study, there were no correlations between the recurrence of endometriosis and previous operations.

As previously discussed, two major risk factors of endometrioma recurrence that were revealed in our study, a younger age at surgery and post-operative dysmenorrhea, could both be explained by theories of endometrioma recurrence. In younger patients, serum estrogen levels are relatively high, which could lead to further stimulation and development of endometrioma from retrograde menstruation after surgery [9]. Equally, on the other hand, postoperative dysmenorrhea could have been caused by residual lesions from previous surgery. However, to further clarify the underlying mechanism of endometrioma recurrence and its associated risk factors, additional studies are warranted.

The recurrence rate of endometrioma after primary surgery followed by postoperative medical treatment for 18 months has been reported to be $19.1 \%$ after 2 years and $40 \%-50 \%$ after 5 years [34]. Several studies reported that the incidence of endometriosis recurrence after laparoscopic surgery is $19-50 \%[13,35]$. We found that the estimated cumulative incidences of recurrence rate were $1.5 \%, 9.7 \%, 13.4 \%, 17.2 \%$, and $26.1 \%$ respectively, at $1,2,3$, 4 , and 5 years after surgery. These relatively low recurrence rates may be due to long-term medical therapy and the recurrence definition applied in our study. Postoperative medical treatment is known to delay the recurrence, but not completely prevent it [36].

Our study has several limitations. The follow-up periods for some of the patients in our population were relatively short, and the study included a very small number of subjects. Also, medical therapy was not identical for all patients, 
which possibly influenced the recurrence rate. However, our study covered patients who were treated with long-term postoperative medical therapy for recurrence prevention. All study subjects received postoperative medical therapy for more than 12 months. Because current studies on endometriosis treatment focus on its medical aspects, it would be meaningful to analyze the factors related to the recurrence rate in those endometriosis patients under a long-term period of medical treatment. Furthermore, this retrospective study was designed to focus correlations between the risk factors of endometriosis recurrence, with less weight given to the underlying mechanism and causal relationships between each of the risk factors and its corresponding statistical results; so further studies regarding such issues are needed.

Furthermore, we studied 18 potential risk factors to identify any clinical patterns in the study subjects. It is very important to predict the risk of recurrent endometrioma to determine postoperative treatment, and we believe our findings are especially helpful for counselling patients at high risk of recurrence.

\section{CONCLUSIONS}

We concluded that dysmenorrhea after surgery, and a younger age at the time of surgery (odds ratio 12.226, $\mathrm{p}$-value $<0.0001$ and $0.924, \mathrm{p}$-value $=0.029$, respectively) were risk factors that are associated with recurrent endometrioma, despite long-term postoperative medication.

\section{Acknowledgements}

This work was supported by the Department of Biostatistics, Clinical Trial Center, Biomedical Research Institute, Pusan National University Hospital. We thank the Department of Biostatistics, Clinical Trial Center, Biomedical Research Institute, Pusan National University Hospital.

\section{REFERENCES}

1. Berkkanoglu M, Arici A. Immunology and endometriosis. Am J Reprod Immunol. 2003; 50(1): 48-59, indexed in Pubmed: 14506928.

2. Chapron $C$, Vercellini $P$, Barakat $H$, et al. Management of ovarian endometriomas. Hum Reprod Update. 2002; 8(6): 591-597, indexed in Pubmed: 12498427.

3. Olive DL, Pritts EA. Treatment of endometriosis. N Engl J Med. 2001; 345(4): 266-275, doi: 10.1056/NEJM200107263450407, indexed in Pubmed: 11474666.

4. Kennedy S, Bergqvist A, Chapron C, et al. ESHRE Special Interest Group for Endometriosis and Endometrium Guideline Development Group. ESHRE guideline for the diagnosis and treatment of endometriosis. Hum Reprod. 2005; 20(10): 2698-2704, doi: 10.1093/humrep/dei135, indexed in Pubmed: 15980014.

5. Macer ML, Taylor HS. Endometriosis and infertility: a review of the pathogenesis and treatment of endometriosis-associated infertility. Obstet Gynecol Clin North Am. 2012; 39(4): 535-549, doi: 10.1016/j. ogc.2012.10.002, indexed in Pubmed: 23182559.

6. Jones KD, Sutton CJ. Laparoscopic management of ovarian endometriomas: a critical review of current practice. Curr Opin Obstet Gynecol. 2000; 12(4): 309-315, indexed in Pubmed: 10954152.

7. Dunselman GAJ, Vermeulen N, Becker C, et al. European Society of Human Reproduction and Embryology. ESHRE guideline: management of women with endometriosis. Hum Reprod. 2014; 29(3): 400-412, doi: 10.1093/humrep/det457, indexed in Pubmed: 24435778.

8. Hart RJ, Hickey $M$, Maouris $P$, et al. Excisional surgery versus ablative surgery for ovarian endometriomata. Cochrane Database of Systematic Reviews. 2005, doi: 10.1002/14651858.cd004992.pub2.

9. Bozdag G. Recurrence of endometriosis: risk factors, mechanisms and biomarkers. Womens Health (Lond). 2015; 11(5): 693-699, doi: 10.2217/whe.15.56, indexed in Pubmed: 26439119.

10. Seo JW, Lee DY, Yoon BK, et al. The age-related recurrence of endometrioma after conservative surgery. Eur J Obstet Gynecol Reprod Biol. 2017; 208: 81-85, doi: 10.1016/j.ejogrb.2016.11.015, indexed in Pubmed: 27894033.

11. Sengoku K, Miyamoto T, Horikawa M, et al. Clinicopathologic risk factors for recurrence of ovarian endometrioma following laparoscopic cystectomy. Acta Obstet Gynecol Scand. 2013; 92(3): 278-284, doi: 10.1111/aogs.12051, indexed in Pubmed: 23194011.

12. Kikuchi I, Takeuchi H, Kitade M, et al. Recurrence rate of endometriomas following a laparoscopic cystectomy. Acta Obstet Gynecol Scand. 2006; 85(9): 1120-1124, doi: 10.1080/00016340600627154, indexed in Pubmed: 16929419.

13. Koga $K$, Osuga $Y$, Takemura $Y$, et al. Recurrence of ovarian endometrioma after laparoscopic excision. Hum Reprod. 2006; 21(8): 2171-2174, doi: 10.1093/humrep/del125, indexed in Pubmed: 16644912.

14. Ouchi N, Akira S, Mine K, et al. Recurrence of ovarian endometrioma after laparoscopic excision: risk factors and prevention. J Obstet Gynaecol Res. 2014; 40(1): 230-236, doi: 10.1111/jog.12164, indexed in Pubmed: 24102958.

15. Lee SY, Kim ML, Seong SJu, et al. Recurrence of Ovarian Endometrioma in Adolescents after Conservative, Laparoscopic Cyst Enucleation. J Pediatr Adolesc Gynecol. 2017; 30(2): 228-233, doi: 10.1016/j.jpag.2015.11.001, indexed in Pubmed: 26612115.

16. Kim ML, Kim JM, Seong SJu, et al. Recurrence of ovarian endometrioma after second-line, conservative, laparoscopic cyst enucleation. Am J Obstet Gynecol. 2014; 210(3): 216.e1-216.e6, doi: 10.1016/j. ajog.2013.11.007, indexed in Pubmed: 24215855.

17. Crosignani $P$, Olive $D$, Bergqvist $A$, et al. Advances in the management of endometriosis: an update for clinicians. Hum Reprod Update. 2006; 12(2): 179-189, doi: 10.1093/humupd/dmi049, indexed in Pubmed: 16280355

18. Timmerman D, Valentin L, Bourne TH, et al. International Ovarian Tumor Analysis (IOTA) Group. Terms, definitions and measurements to describe the sonographic features of adnexal tumors: a consensus opinion from the International Ovarian Tumor Analysis (IOTA) Group. Ultrasound Obstet Gynecol. 2000; 16(5): 500-505, doi: 10.1046/j.1469-0705.2000.0 0287.x, indexed in Pubmed: 11169340.

19. Revised American Society for Reproductive Medicine classification of endometriosis: 1996. Fertil Steril. 1997; 67(5): 817-821, indexed in Pubmed: 9130884.

20. Porpora MG, Pallante $D$, Ferro $A$, et al. Pain and ovarian endometrioma recurrence after laparoscopic treatment of endometriosis: a long-term prospective study. Fertil Steril. 2010; 93(3): 716-721, doi: 10.1016/j. fertnstert.2008.10.018, indexed in Pubmed: 19061997.

21. Tandoi I, Somigliana E, Riparini J, et al. High rate of endometriosis recurrence in young women. J Pediatr Adolesc Gynecol. 2011; 24(6): 376-379, doi: 10.1016/j.jpag.2011.06.012, indexed in Pubmed: 21906976.

22. Liu X, Yuan L, Shen F, et al. Patterns of and risk factors for recurrence in women with ovarian endometriomas. Obstet Gynecol. 2007; 109(6): 1411-1420, doi: 10.1097/01.AOG.0000265215.87717.8b, indexed in Pubmed: 17540815.

23. Parazzini F, Bertulessi C, Pasini A, et al. Gruppo Italiano di Studio Endometriosi. Determinants of short term recurrence rate of endometriosis. Eur J Obstet Gynecol Reprod Biol. 2005; 121(2): 216-219, doi: 10.1016/j. ejogrb.2004.11.033, indexed in Pubmed: 16054965.

24. Hediger ML, Hartnett HJ, Louis GM. Association of endometriosis with body size and figure. Fertil Steril. 2005; 84(5): 1366-1374, doi: 10.1016/j. fertnstert.2005.05.029, indexed in Pubmed: 16275231.

25. Liu Y, Zhang W. Association between body mass index and endometriosis risk: a meta-analysis. Oncotarget. 2017; 8(29), doi: 10.18632/oncotarget.14916.

26. Tobiume $\mathrm{T}$, Kotani $\mathrm{Y}$, Takaya $\mathrm{H}$, et al. Determinant factors of postoperative recurrence of endometriosis: difference between endometrioma and pain. Eur J Obstet Gynecol Reprod Biol. 2016; 205: 54-59, doi: 10.1016/j. ejogrb.2016.07.516, indexed in Pubmed: 27566223.

27. Harada $T$, lida $T$, Chikamura $C$, et al. Factors predicting bone mineral density (BMD) changes in young women over a one-year study:changes in body weight and bone metabolic markers during the menstrual cycle 
and their effects on BMD. Acta Med Okayama. 2012;66(4): 307-315, doi: 10.18926/AMO/48670, indexed in Pubmed: 22918203.

28. Ashrafi M, Sadatmahalleh SJ, Akhoond MR, et al. Evaluation of Risk Factors Associated with Endometriosis in Infertile Women. Int J Fertil Steril. 2016; 10(1): 11-21, indexed in Pubmed: 27123195.

29. Nominato NS, Prates LF, Lauar I, et al. Caesarean section greatly increases risk of scar endometriosis. Eur J Obstet Gynecol Reprod Biol. 2010; 152(1): 83-85, doi: 10.1016/j.ejogrb.2010.05.001, indexed in Pubmed: 20510495.

30. AndolfE, Thorsell M, Källén K. Caesarean section and risk for endometriosis: a prospective cohort study of Swedish registries. BJOG. 2013; 120(9): 10611065, doi: 10.1111/1471-0528.12236, indexed in Pubmed: 23663181.

31. Mavrelos D, Saridogan E. Treatment of endometriosis in women desiring fertility. J Obstet Gynaecol India. 2015; 65(1): 11-16, doi: 10.1007/s13224014-0652-y, indexed in Pubmed: 25737616.
32. Taff $\mathrm{L}$, Jones $\mathrm{S}$. Cesarean scar endometriosis. A report of two cases. J Reprod Med. 2002; 47(1): 50-52, indexed in Pubmed: 11838312.

33. Sengul I, Sengul D, Kahyaoglu $S$, et al. Incisional endometriosis: a report of 3 cases. Can J Surg. 2009; 52(5): 444-445, indexed in Pubmed: 19865584.

34. Guo SW. Recurrence of endometriosis and its control. Hum Reprod Update. 2009; 15(4): 441-461, doi: 10.1093/humupd/dmp007, indexed in Pubmed: 19279046.

35. Busacca M, Marana R, Caruana P, et al. Recurrence of ovarian endometrioma after laparoscopic excision. Am J Obstet Gynecol. 1999; 180(3 Pt 1): 519-523, indexed in Pubmed: 10076121.

36. Berlanda N, Morini M, Dridi D, et al. Effect of Long-Term Use of Hormones on Endometriomas. Current Obstetrics and Gynecology Reports. 2013; 2(3): 178-185, doi: 10.1007/s13669-013-0053-8. 